\title{
Cycles of Diversification in Urban Environ- ments: Evidence from Sassi, UNESCO World Heritage Site, Italy
}

\section{Authors}

Dan C. Baciu, ${ }^{1}$ Diana Della Pietra. ${ }^{23}$

Faculty of Architecture and the Built Environment, TU Delft, Delft, Netherlands.

\section{Lead}

Quantitative evidence from the Italian UNESCO world heritage site of Sassi supports that urban environments go through cycles of diversification and growth.

\section{Abstract}

Evolution goes through cycles of diversification and growth. Here, we report empirical evidence to support this thesis in urbanism. Our research broadens theoretical work on evolutionary theory and breaks new ground for an entire range of applications in the precise planning and management of urban environments and their architecture.

\section{Main text}

Almost every present-day definition of "city" is about urban density and diversity (Dijkstra, Poelman 2012, Maunier 1910, Simmel 1903), and cities have been places of diversity since time immemorial: Babel is known for its languages, Medieval cities for their guilds, and modern cities for cultural diversity. It is true that Babel also had a tower, Medieval cities had walls, and modern cities have electrical grids, but these infrastructures are changing, while diversity stays.

Here, we study how diversity forms, theorizing that it tends to evolve in cycles of diversification and growth: Diversity catalyzes growth, but growth comes with competitive exclusion, which reduces diversity. Cycles of diversification and growth have

\footnotetext{
1 https://orcid.org/0000-0002-0043-5616

2 https://orcid.org/0000-0001-7373-8901

${ }^{3}$ Both co-authors have contributed equally to this paper.
} 
previously been shown to exist in human culture, where we have modeled them with the replicator-mutator-equation (Baciu 2020).

Looking for evidence to test this theory and make predictions in urbanism, we found that famous urban activist Jane Jacobs has already written, in the 1960s, an entire chapter titled the "Self-destruction of diversity" in which she provided empirical observations that very closely match our theoretical predictions. She observed that growth reduces diversity. Her chapter is part of the book "The Death and Life of Great American Cities," which spurred a flurry of subsequent activism and research (Quiqley 1998).

Our predictions come with more detail and a deeper theoretical and mathematical foundation. The theory that we would like to test says that there are cycles of growth and diversification that can be modeled with the replicator-mutator-equation. We predict that there are two phases, diversification and growth. Each phase corresponds to one parameter region of the replicator-mutator-equation: Diversification corresponds to the replicator part of the equation, while growth corresponds to the mutator part (Eqn. 1). In their everyday workings, the two parts tend to fall apart, and we have distinct phases of diversification and growth. Of course, it might be beneficial to keep the equation together, avoiding the cycles, and building a straight line to success.

Equation 1: The replicator-mutator-equation states that each idea in new culture $\dot{x}_{i}$ is the product of all existing culture $x_{1 \ldots n}$, creativity $q_{j i}$, and interplay $f_{j}(\mathrm{x})$. The creative part of the equation $q_{j i}$ describes how ideas cluster into larger adaptive groups. The groups are held together by creativity and can be imagined as creative clouds or creative swarms. People observe and classify such groups, often calling them 'categories' or 'types.' As they grow, these groups compete against each other, which leads to competitive exclusion. The interplay part of the equation $f_{j}(\mathrm{x})$ has two modes of action. It can further increase competitive exclusion, or it can adjust the growth rates $f_{j}$ in such way as to alleviate competitive exclusion and allow for diversification. This latter adjustment mostly leads to an overall reduction of growth. (When you steer a boat, it goes slower.) As a result, periods of diversification are periods of reorientation-and often of recession. In human culture, the switch between growth and diversification is controlled by the human perception of diversity (Baciu 2020, 2021). The replicator-mutator-equation was first formulated in the life sciences. There, the creative part of the equation is known as the mutator parameter region while the interplay part is known as replicator parameter region. Hence the name, replicator-mutator-equation. For this earlier treatment see Page \& Nowak 2001.

$$
\dot{x}_{i}=\sum_{j=1}^{n} x_{j} q_{j i} f_{j}(\mathrm{x})
$$

Jane Jacobs's observations provide a first empirical proof that cycles of diversification actually exist in urban environments. However, we wished to obtain more proofs. In particular, we wished more quantitative, replicable, and more up-to-date evidence. In a first article (Baciu, Birchall 2021), we developed a method to quantify urban 
diversity. We felt that, given that cities are defined as places of diversity, such a method would be immensely useful. We succeeded in demonstrating that our mapping works, and that it can be used to make forecasts, evaluating how diversity changes with every single intervention that planners envision in the urban environment. We now employ the same method, but in a longitudinal study that stretches over several decades (technical details and links to data in conjoint MethodsX-paper).

Given that we are engaging in a longitudinal study, the historical quarter of Sassi in the Italian city of Matera seemed an appropriate choice (Fig. 1). Sassi's diverse history is well documented through maps, text, and photographs. Let us begin by portraying the quarter through a beautiful historical description. Already a century ago, Sassi was perceived as a picturesque place of diversity in terms of both architectural form and human activity. A newspaper article reads, Sassi "is a continuous variety of light and shade, a picturesque game of color, a medley of streets, parapets, arches, window sills, gables, balconies, corbels, and galleries in which it is useless to seek symmetry, alignment, or order. [...] In this truly beautiful and varied pictureframe, human activity adds an even more beautiful tune" (Calamonico 1927).
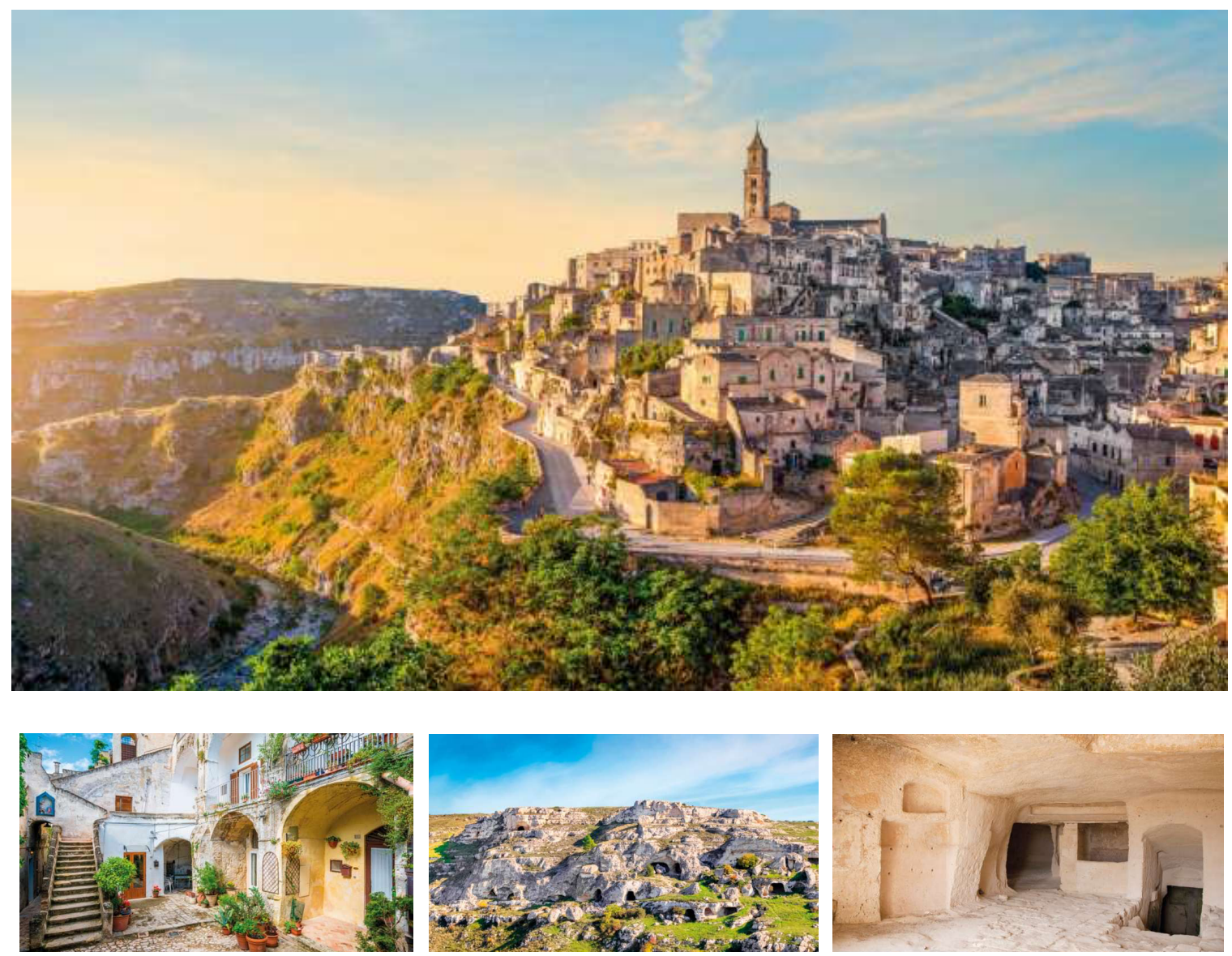

Figure 1. "Matera, the Rebirth of the City of the Rocks." Touristic presentation of Sassi by Costa Crociere (2018). Sassi literally means "rocks". This name comes from both the beautiful landforms and ancient cave dwellings that have been partly preserved. The city's annals document a continuing history of diversification and growth. 
Using historical records, we quantified urban diversity in Sassi over the last five decades, and we validated our results, this time through three independent methods. Among others, the validation also showed us that diverse areas retained their inhabitants or have grown, and that they formed urban centers (details in MethodsX-paper). Now that we are confident that our maps are good estimates of urban diversity, we are ready to interpret our observations. What we see is a history that we, and Jane Jacobs, could only have dreamt of. We see how Sassi naturally runs through a cycle of diversification and growth, and how the population was torn between enhancing or limiting the effects of this cycle through legislation (Fig. 2).

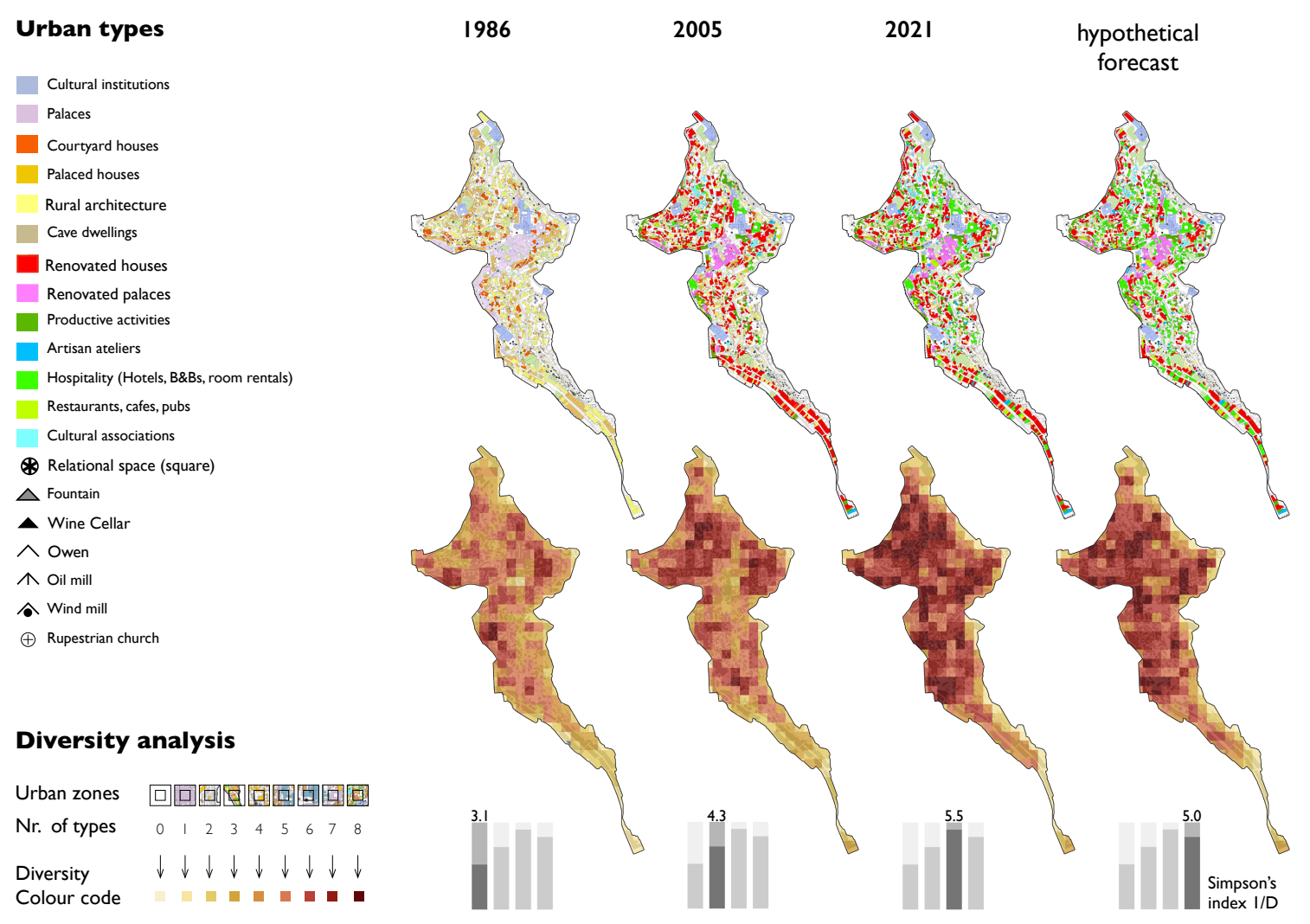

Figure 2. Diversity maps of Sassi. In the period 1986-2021, Sassi has become UNESCO world heritage (1993), and Matera, the city in which Sassi is located, was elected European cultural capital of 2019. This development has supported a high growth rate in tourism. Between 1986 and 2021, the number of hotels has doubled several times over (hospitality venues marked bright green on maps; growth also documented by Toxey 2011). This hotel expansion has initially contributed to diversification. A touristic advertisement playfully nicknamed Sassi a "rebirth" (Fig. 1), and officials wrote that Sassi "returned to life" (MethodsX). Nevertheless, as illustrated in our hypothetical forecast, a further doubling in the number of hotels would reduce diversity. Jane Jacobs may have liked seeing this particular forecast when she wrote, "The duplication of the most profitable use is undermining the base of its own attraction, as disproportionate duplication and exaggeration of some single use always does in cities" (Jacobs, 1961, p. 245). What is new is that we can now quantify and simulate when precisely hotel expansion enhances and when it reduces diversity. This forecasting ability will make it possible to manage urban environments with a theoretically unlimited precision at any resolution in both space and time. 
Present-day legislation in Matera proves just how difficult it has been to sustainably manage diversification and growth. In 2016, as Matera's tourism flourished, a new law was enacted that would have facilitated further growth in the tourism industry. This law was practically reversed in Sassi when immediate protection of many buildings followed. The city and its inhabitants figured that they had to protect Sassi's diversity, even if this meant limiting growth.

The legislation that was enacted in Matera has a double effect. The law supports tourism everywhere, except in the old center of Sassi where the law is reversed through massive individual protection of buildings. The reason for this uneven treatment is that tourism still contributes to diversification in most places, although it reduces diversity in Sassi. The complexities of urban space led to complications in legislation.

There is another, easier way to formulate law that achieves the same goals. The law can simply set goals for diversification. At every point in space and time, the same question is asked again and again: How can the diversity goals be achieved? The response may vary with local conditions at any given point in time. We call this type of management "precise management." With our mapping method, it is possible to unambiguously translate unchanging law into precise planning and management (MethodsX-paper). In addition, an early warning system could be set up to automatically detect lack of diversity or lagging growth in any urban area, thus helping local populations launch more specifically targeted efforts to level disruptive cycles of diversification.

\section{When diversity is not planned: The dark history of Sassi}

Sassi's history also documents a dark and sad decade that we wished to understand. Everything looked fine in the 1930s, only years after the above-quoted newspaper described Sassi as a picturesque place. City life was lively. New fountains replaced an older system of underground cisterns and provided additional public space for gatherings and urban activities. However, only two decades later, in the 1950s, the inhabitants of Sassi were abruptly relocated into modern suburbs. Planners were suddenly convinced that modernity was better than tradition.

The ensuing history proves that the planners were wrong. Sassi was revived, and its rebirth was celebrated among other by the tourism industry (Fig 1.). On the other hand, the modern suburbs beg for attention, humiliated by repeated episodes of vandalism, sporadic demolitions, and years of neglected maintenance (Saito 2020).

Our evaluation shows that there is a notable difference between Sassi and the modern suburbs: The first is polycentric, it teems with diversity, and it retained its inhabitants. The latter are monocentric, lack diversity, and some inhabitants are reported to have returned to Sassi (Fig. 3). This evidence not only reveals the tragedy of past mismanagement but also supports the thesis that lack of diversity may result in decay. 


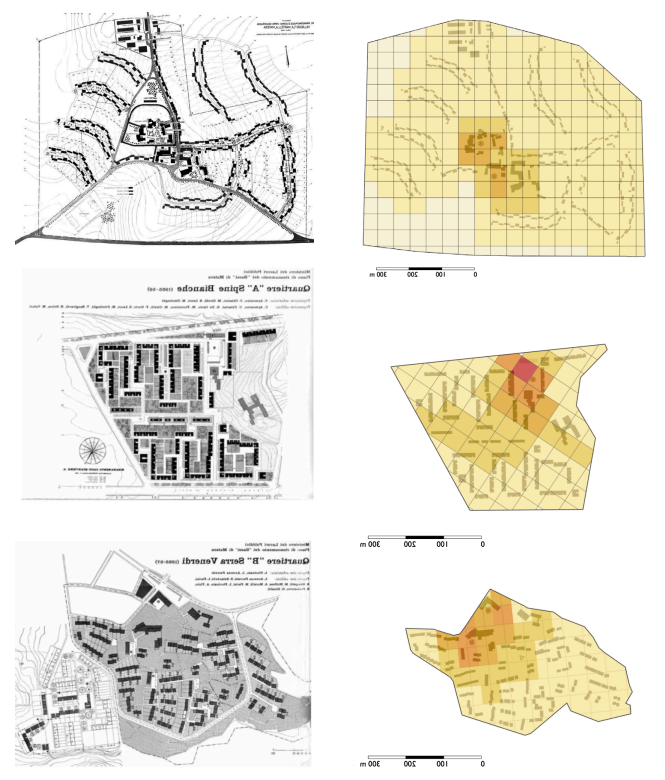

Figure 3. In the 1950s, the inhabitants of Sassi were forcedly relocated into modern suburbs. However, these suburbs were not planned to be diverse and provided only minor centers of urban diversity. Some of the displaced inhabitants eventually returned to Sassi. For technical details see conjoint MethodsX-paper.

Thus, over the course of this article, we have provided empirical evidence to support not only that diversity can catalyze growth and that diversification may go through cycles of diversification and growth, but also that lack of diversity may be paired with decay.

\section{Discussion}

Studying the UNESCO world heritage site of Sassi, we have found empirical evidence to support the thesis that urban environments go through cycles of diversification and growth. Using a new method and theory, we are now able to quantify and simulate these cycles.

The maps that we provided in our empirical evaluation display fine-grained resolution, and our mapping method allows for assessment and reassessment of existing conditions in space and time, as well as hypothetical simulation of the effects that any planned urban intervention could have on urban diversity. Because of these properties, our maps and methods can facilitate precise planning and management. Authorities and citizen alike can now easily and unambiguously translate binding and unchanging law into tailored planning strategies in response to local or simulated conditions: The law is the same for everyone, the effects may vary. This breaks the ground for an entire range of applications in the precise planning and management of urban environments and their architecture.

Our insights come from modern Sassi, but they are not limited to this particular epoch or place. In the bigger picture, we see a recurrent pattern. Great infrastructures (Babylonian towers, Medieval fortresses, and modern electrical grids) and dominant urban activities (commerce, cultural tourism) one after the other grow out of the diversity of urban life only to eventually return into it. This same recurrent pattern is also found other fields of study. In cultural studies, dominant mainstreams grow out of the 
diversity of cultural life to eventually return into it; and in virus dynamics, acute infections in individual patients challenged with persistent diseases, or pandemics in entire populations, grow out of viral diversity only to eventually return into it. This "cycle of diversification and growth" can be described and explained in all of these disciplines with the replicator-mutator-equation or variants and special cases thereof.

\section{MethodsX}

Our MethodsX-paper published in conjunction with this present article lays down the methodological details necessary for replication. In particular, the MethodsX-paper gives special attention to the methodological customizations made for the present longitudinal study of urban diversity. Otherwise, the paper follows the six steps towards the evaluation of diversity laid out by Baciu \& Birchall (2021): First, we define 'diversity.' Second, we specify what record is employed in the analysis. Third, we lay down how diversity is to be evaluated through the choice of a classification system, granularity, and diversity index. Fourth, we detail the mapping procedure. Fifth, we validate the results. Finally, we propose potential applications of our method in the management and planning of urban environments. 


\section{Bibliography}

Baciu DC (2020). "Cultural life: Theory and empirical testing." BioSystems 197.104208.

Baciu DC (2021). "Digital life: Creative and open-ended." OSF Preprints DOI: 10.31219/osf.io/hje26

Baciu DC, Birchall C (2021). "Mapping Diversity: From Ecology and Human Geography to Urbanism and Culture." OSF preprints.

Costa Crociere (2018) "Matera, la rinascita della città dei Sassi." Online story, retrieved from www.costacrociere.it 20.04.2021.

Dijkstra L, H Poelman (2012). "Cities in Europe: The New OECD-EC Definition" Regional Focus 01/2012.

Fisher RA, AS Corbet, CB Williams (1943). "The Relation Between the Number of Species and the Number of Individuals in a Random Sample of an Animal Population." J. Animal Ecol., 12, 42.

Hirschman AO (1945). National power and the structure of foreign trade. Berkeley: University of California Press.

Jacobs J (1961). The Death and Life of Great American Cities. New York: Random House.

Maunier R (1910). "The Definition of the City.” American Journal of Sociology 15, 536548.

Page K, Nowak MA (2002). “Unifying Evolutionary Dynamics.” J. theor. Biol. 219, 9398.

Saito M (2020) Press Release. Do.Co.Mo.Mo Italia March 18. 2020.

Simmel G (1903). "Die Großstädte und das Geistesleben." Die Großstadt: Vorträge und Aufsätze zur Städteausstellung, Dresden: Gehe-Stiftung, 185-206.

Toxey A (2011). Materan Contradictions: Architecture, Preservation and Politics (Ashgate Studies in Architecture). Ashgate Publishing Group.

Quigley JM (1998).“Urban Diversity and Economic Growth.” Journal of Economic Perspectives 12.2: 127- 138.

\section{Contributions}

Both authors contributed equally to this research. Dan C. Baciu has written the article. Diana Della Pietra has studied urban diversity and created the diversity maps of Matera as student under the lead of Dan C. Baciu as Assistant Professor of Digital Tools at TU Delft, Faculty of Architecture and the Built Environment. 


\section{MethodsX-paper for: Cycles of Diversifica- tion in Urban Environments: Evidence from Sassi, UNESCO World Heritage Site, Italy}

\section{Authors}

Dan C. Baciu, ${ }^{4}$ Diana Della Pietra. ${ }^{56}$

Faculty of Architecture and the Built Environment, TU Delft, Delft, Netherlands.

\section{Abstract}

In the Italian UNESCO world heritage site of Sassi, we studied urban diversification and growth, covering a period of five decades. This present MethodsX-paper contains the methodological considerations and specifications. The research results that we obtained through this evaluation are reported in our research article "Cycles of Diversification in Urban Environments" (Baciu, Della Pietra 2021).

\section{Main text}

In an earlier methods-paper (Baciu, Birchall 2021), we have demonstrated how to analyze and map diversity in urban environments. Here, we follow the same method. The methodological customizations that we have undertaken stem primarily from the fact that our present study is longitudinal, covering five decades. These customizations are marginal, in general. Our previously developed methodology has remained mostly unchanged. When explaining the new customizations and laying down the specifications necessary for research reproduction we shall therefore adhere to the six steps towards the analysis and mapping of diversity laid down in our earlier methods-paper:

- First, we define 'diversity.'

- Second, we specify what record is employed in the analysis.

- Third, we lay down how diversity is to be analyzed through the choice of a classification system, granularity, and diversity index.

- Fourth, we detail the mapping procedure.

- Fifth, we validate the results.

- Finally, we propose applications of our method in the precise management and planning of urban environments.

\footnotetext{
4 https://orcid.org/0000-0002-0043-5616

5 https://orcid.org/0000-0001-7373-8901

${ }^{6}$ Both co-authors have contributed equally to this paper.
} 


\section{Defining diversity}

In general, diversity is "the probability that different parties meet." This definition was first employed in ecology and linguistics (Fisher 1943, Yule 1944, Simpson 1949), yet, it was independently formulated in economics (Hirschman 1945), and it found applications in politics (there known as effective number of parties), in physics (there known as inverse participation ratio), in virology (Nowak 1991), in the humanities (Baciu 2020), and in urbanism (Baciu, Birchall 2021).

It is logical that diversity, as defined above, must be a defining characteristic of cities: Cities are centers of urban interaction, and interaction makes most sense between different parties. Same parties have fewer reasons to meet simply because they cannot complement each other as well. Thus, cities are places where different people meet and interact.

At this point, it may be relevant to define "interactions." We shall consider as an interaction everything that people do in a city. In our previous article (Baciu, Birchall 2021), we listed some examples. Interactions are things such as: Meeting friends at a bar; doing the groceries; shopping; returning home; or going to a restaurant, a sports venue, a beauty salon, a cultural event, a congregation, etc. These activities are all interactions because they cannot be done in isolation. The list can remain open-ended at this point, but in our final evaluation of urban diversity, we would like to consider as many as possible such interactions.

Just what makes people, activities, and urban environments "different" or, more precisely, "diverse" may be equally important to define. Given that we work with the replicator-mutator-equation, we study two main processes: creativity and diversification. Together, these two processes lead to the formation of diverse types. Within the types, there is creativity, between them, there is diversification (Baciu 2020). This leaves us with the insight that each type consists of creative interpretations and reinterpretations. For example: Most hotels are creative reinterpretations of other hotels, while artisan ateliers are most frequently reinterpretations of other artisan ateliers. On this basis diverse urban types such as hotels and artisan ateliers evolve naturally; they form natural clusters.

On this basis, our definition of urban diversity requires a multitude of environments in which and with which people interact. For example, meeting friends at a bar requires a bar, while making the groceries requires a grocery. Thus, our definition of urban diversity comes close to what urban planners call "mixed use." The main difference is that we look from the perspective of people who engage in diverse types of interactions, while planners look from the perspective of builders who create diverse types of buildings in which or with which people can interact. The similarity between our definition of diversity and mixed use will is practical when operationalizing the research idea. There is no reliable record of people going to bars, but there usually is reliable record of where bars are located. 
It is sometimes useful to imagine diverse types each as a distinct creative cloud or creative swarm of ideas. The precise shape of each swarm is a steady-state equilibrium between creativity that stretches the swarm in all directions and selection that narrows it in. Such creative swarms form units of cultural evolution. They can be modeled with the replicator-mutator-equation or mathematical equivalents thereof (Baciu 2020). Creative swarms make up the backbone of the diversity in anything from social fields, to human language, human culture, as well as biological evolution (Baciu 2020). In molecular genetics, they are also referred to as quasispecies.

In some cases, it is possible to study diverse types through computer-driven techniques of dimensionality reduction. These techniques simulate the mutator part of the replicator-mutator equation (Baciu 2020). The results obtained from the computer-driven analysis are estimates and must usually be validated through human inspection: The analysis is considered valid if humans recognize diverse types in it.

In other cases, dimensionality reduction may not be needed at all. People typically categorize the world into diverse types of the kind we wish to study. If a detailed human analysis is available, there is almost no reason to look any further. In particular in longitudinal studies like ours, human analysis is desirable because dimensionality reductions are not good at simulating changing conditions and adaptation.

We have chosen this definition of diversity because it is in line with human perception. It is also in line with our mathematics, and it can be translated to many other disciplines.

\section{The record employed in the analysis}

To come as close as possible to the above-given definition of urban diversity, we choose to study the UNESCO world heritage site of Sassi, Italy. In 1993, Sassi has been listed as heritage site of universal value. This made us trust that the records created around this period have been processed with care and are intended to well represent human activities, interactions, and modes of life. From the governmental documents created during the renovation of Sassi (Commune di Matera 2005, 4), we read that the authorities that surveyed it intended to meticulously record diverse urban "types" and urban "life", past and present:

'The restoration of Sassi does not end when the last building has been renovated and preserved because [Sassi's] 'return to life' brings new functions and new typological interpretations; and it is the responsibility of the community to make new uses compatible with [Sassi's] millennial history. [...] The maps sum up what has been studied and concluded in the space of twenty years on the urban settlement of Sassi. [...] These maps are therefore an ideal 'witness' of the continuity of the cultural framework which is at the foundation [our survey]. Today, [in 2005,] when most of the preservation efforts show tangible results, these maps can provide a useful tool to evaluate the interventions that have so far been realised [...] and to shape future plans.' 
Reading this and other similar excerpts, it is evident that the record available for Sassi and the framework employed there to categorize urban infrastructures into different urban types 1 ) represent a well-developed system of classification into diverse urban types, while 2) leaving room for reinterpretation and creativity. In particular, the officials write that urban types are open to constant "typological interpretation." An urban type thus comes close to a cloud of creative interpretations and reinterpretations, just as we required in section 1 . We should mention that this good match for us is no coincidence. People naturally categorize the world around them into diverse types, just as it has been done in Sassi.

At this point, we should mention that making a good estimate of diversity does not require a complete but an accurate record of the urban environment. Evidently, some urban activities and interactions are very hard to record, which makes the record accordingly unreliable, yet unreliable records are detrimental for good estimates. Thus, we require reliable and accurate data, which makes Sassi a particularly good example to work with, because of the analytical care with which the records were compiled. These records are reliable because they are part of a longstanding management and preservation strategy for a world heritage site of universal value. The records were compiled not only as an "ideal witness" that represents the past, but they are also meant to be helpful in "shaping future plans," as the officials write in the above-given quotation.

Given this outstanding quality of the records, we base our own evaluation primarily on the governmental survey that began in 1986 (Commune di Matera 2005, 4). Sassi had just been resettled, but renovation had not yet started. Just then, a law was passed that ordered records to be made to document the state of the urban environment at that point in time. From 1986 onward, the records available to us remain accurate and valuable. Therefore, we choose 1986 as our starting point.

The record made beginning with 1986 is particularly useful for our longitudinal study because the survey that began in 1986 itself stretched over many decades, and experience was accumulated. In 2005, two maps were drawn that documented how Sassi had changed. One map represents the state of the urban environment in 1986 (Commune di Matera 2005, 1), while the other represents the new interventions made between 1986 and 2005 (Commune di Matera 2005, 2). The 1986-map was meant to document the pre-existing situation, while the 2005-map focused on new interventions alone. These two maps serve our goal perfectly because we intend to distinguish between two distinct periods of time, just as it is done on the two maps. The maps help us geospatially trace how new urban types were introduced between 1986 and 2005.

Following these considerations, we used the 1986-map with only minor changes: We added fountains, which had been omitted on the original plan. We then created our "2005"-map by overlaying the two maps 1986 and 2005 (Commune di Matera $2005,1-2$ ). In doing so, we chose the 1986-map as a base-map and superimposed the 2005 map. Wherever buildings were used in new ways in 2005, we recorded this new usage on our 2005-map. Thus, newly introduced urban types replace older urban types. 
To obtain our "2021"-map, we further updated our 2005 map by superimposing official geocoded records for April 2021 (Ufficio Statistica 2021, 2), as well as 2021 google maps data. Given that the official records are used to manage the city and are updated on a biweekly basis, and given that google maps are commonly used to navigate the city, we hold the combination of these two sets of data to be sufficiently complete for our present purpose.

We should note that our 2021-map may be useful to other planners in Matera. The map is a re-evaluation of the present situation that was long due. While the Statistics Office of the City of Matera dutifully collected most of the necessary data for this map, nobody undertook to update the maps from 1986 and 2005. Our map represents such a long-due update.

Finally, our "hypothetical forecast" is obtained by once again updating the 2021map. To make the forecast replicable, we chose to double the number of hotels by placing a new hotel south and adjacent to each existing hotel.

Next to Sassi, we also evaluate the following modernist settlements created in the second part of the $20^{\text {th }}$ century: La Martella, Borgo Venusio, Spine Bianche, and La Nera. The modernist settlements were built from scratch based on new architectural plans. These are the plans that we used in our evaluation-the particular plans we worked with are printed as such in the research article. Additional information of how the buildings were used is available from the planning records.

\section{Analysis}

Any analysis of diversity has the goal of turning the record under consideration into precise diversity scores. In our previous article (Baciu, Birchall 2021), we found that this processing requires three main choices to be made:

- First, a system of classification must be chosen that lays down which particular categories of things are considered to be diverse. For example, "going to a bar" and "going to a restaurant" can be considered to be the same type of activity, or they can be considered to be different types of activity.

- Second, any analysis must specify on which granularity one wishes to analyze diversity: Does one look at buildings, streets, or entire urban areas?

- Third, it is relevant to specify what formula is to be used to rank different levels of diversity.

As already mentioned, we have chosen Sassi for our present research because the records available document the diversity of urban activities well. When we have chosen these records, we have also chosen the system of classification. The records come with their own system of classification, which, as discussed in section 2, matches our requirements very well (Commune di Matera 2005, 3). For the reader's convenience, the classification system is given in Fig. 2 of the research article. 
Next to choosing a classification system, we must also choose a granularity, which specifies the amount of detail considered in our evaluation of diversity. We use the same granularity as in our previous methods-paper (Baciu, Birchall 2021). We are looking at individual buildings. Each building may represent at most one type of urban activity. Where multiple types are found in one building, the most recent is chosen in the analysis. In addition to buildings, we also consider infrastructures such as fountains, ovens, etc. We employ the same granularity throughout the fifty-year period. Annexed to our MethodsX-paper is a map that exactly defines the granularity that we worked with. Each separate symbol on this map was considered as one item with weight of 1 towards our diversity calculations (Data: Granularity Map, GM).

Finally, we must choose a way to quantify how much diversity is actually found in each area. This choice is also identical with our previous methods-paper (Baciu, Birchall, 2021): Our diversity index values are obtained by overlaying the map with a 100x100m grid, and counting how many different urban types are found in each field of the grid. This indexing is equivalent to "richness" in ecology. Next to this value, we have also calculated Simpson's diversity for Sassi in its entirety. The Simpson index is obtained by counting the total number of items of each urban type in each time period and applying Simpson's formula as 1/D (Eqn. M1). The computations that were performed are shown below the equation. We use the Simpson index to trace how Sassi diversified overall, and we also forecast the effects of the doubling of the number of hotels by numerical simulation. Both through mapping and numerical simulation, diversity decreases in the hypothetical forecast.

Equation M1. We calculate Simpson's index, 1/D, which represents the probability that a person randomly picks two different urban types in a row. $N$ represents the total number of buildings and infrastructures, while $n$ represents the number of infrastructures of each type, specifically.

$$
D=\sum\left(\frac{n}{N}\right)^{2}
$$

\begin{tabular}{|l|r|r|r|r|}
\hline Rural architecture & 1986 & 2005 & 2021 & $\begin{array}{l}\text { Hypothetical } \\
\text { Forecast }\end{array}$ \\
\hline Palaced houses & 1053 & 463 & 235 & 192 \\
\hline Courtyard houses & 759 & 196 & 135 & 111 \\
\hline Wine cellars & 287 & 119 & 54 & 44 \\
\hline Cave dwellings & 90 & 90 & 90 & 74 \\
\hline Palaces & 66 & 60 & 57 & 47 \\
\hline Cultural institutions & 44 & 5 & 5 & 4 \\
\hline Ovens & 22 & 45 & 55 & 45 \\
\hline Fountains & 15 & 15 & 15 & 13 \\
\hline Squares & 13 & 13 & 13 & 11 \\
\hline Oil mills & 7 & 7 & 7 & 6 \\
\hline Wind mills & 4 & 4 & 4 & 3 \\
\hline Renovated houses & 1 & 1 & 1 & 1 \\
\hline Hospitality & 0 & 1017 & 938 & 769 \\
\hline Productive activities & 0 & 58 & 398 & 796 \\
\hline Restaurants, café, pubs & 0 & 168 & 259 & 212 \\
\hline Cultural associations & 0 & 56 & 125 & 103 \\
\hline Artisan ateliers & 0 & 53 & 93 & 76 \\
\hline Renovated Palaces & 0 & 16 & 82 & 67 \\
\hline Simpson's Index 1/D & 0 & 37 & 37 & 30 \\
\hline
\end{tabular}

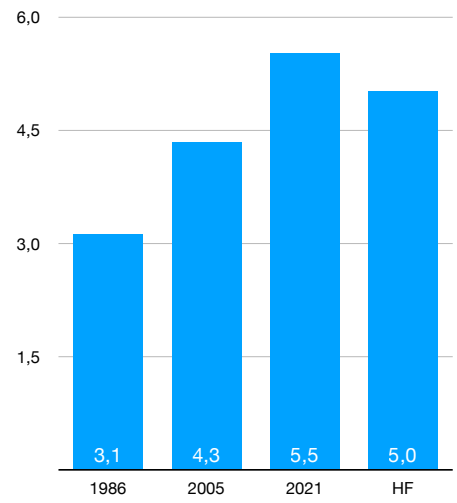

Simpson's Index 1/D 


\section{Mapping}

Although we have chosen a to calculate diversity based on a $100 \times 100 \mathrm{~m}$ analysisgrid, we would like to get a little more resolution on the actual map. To achieve this, we combine the $100 \times 100 \mathrm{~m}$ analysis-grid with a $50 \times 50 \mathrm{~m}$ resolution grid. Each field of $50 \times 50 \mathrm{~m}$ is ranked according to the number of distinct urban types found in the entire $100 \times 100 \mathrm{~m}$ area surrounding and containing the 50x50m field (Fig. M1).

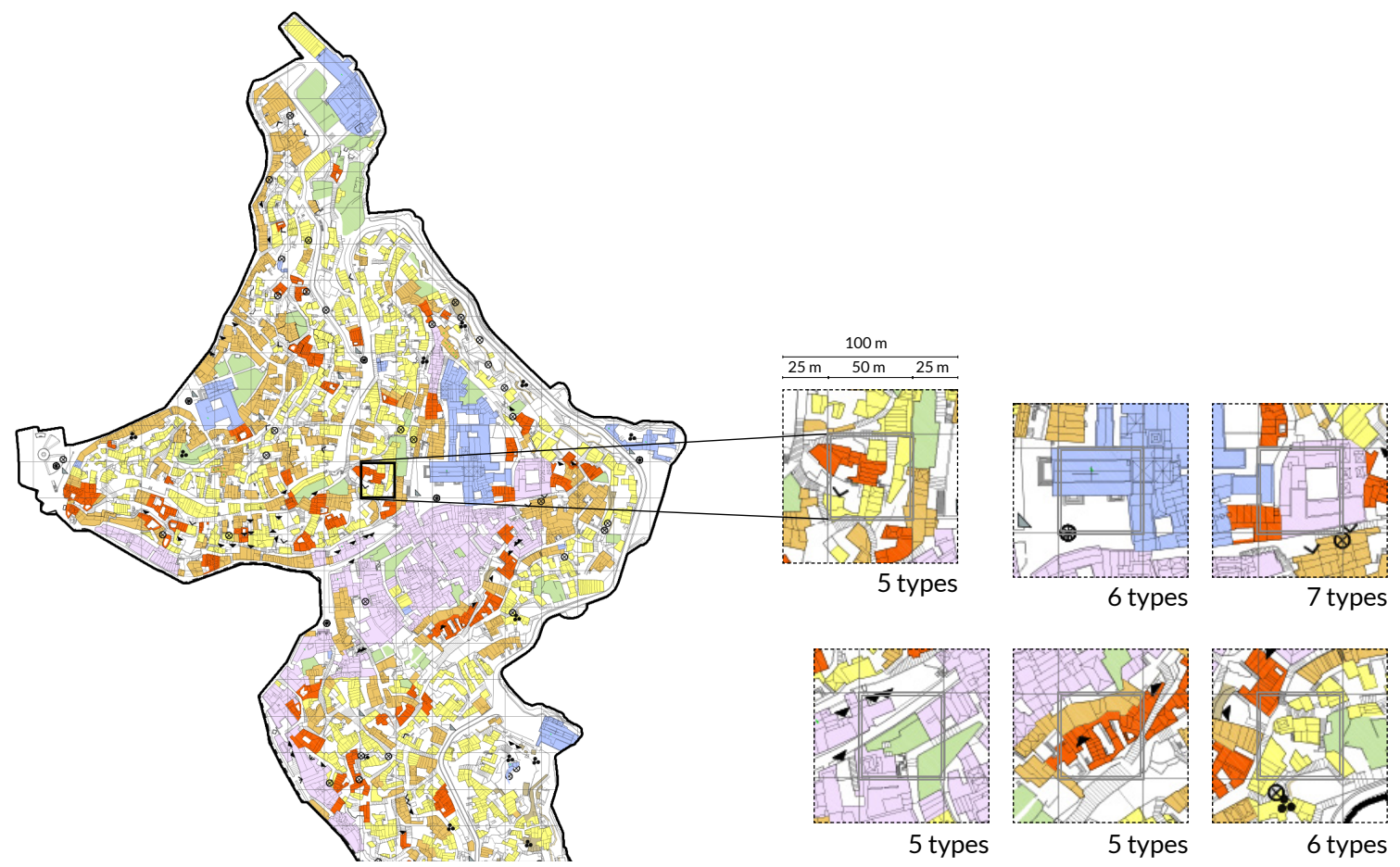

Figure M1. Mapping diversity. The resolution chosen for the maps is a 50x50m grid. The index values are obtained on a slightly larger grid of $100 \times 100 \mathrm{~m}$. The larger grid is chosen because it performed well in our previous research, where it represented well how much of the city people perceive at the same time, on average.

\section{Validation}

By now, we have defined diversity, chosen a record to base our analysis on. Furthermore, we have specified how the analysis is to be performed, and how the results are to be mapped. These choices are logical. In theory, our analysis and mapping should work. Nevertheless, we must doublecheck whether the mapping works not only in theory but also in practice. Let us therefore test whether the results that we obtain are good estimates of diversity. In our earlier methods-paper (Baciu, Birchall 2021), we called this step "validation." Reliable validation is best obtained through paths independent from the method used to create the results. We shall validate our mapping through three independent paths. 
First, we look at the relationship between diversification and growth. When modeling diversification with the replicator-mutator-equation, we suggested that diversity catalyzes growth (Baciu 2020). This insight can be used to test how good our estimates of diversity are. If diversity on our maps stimulates growth, then it should be possible to document this growth through other sources. We look into census data and identify which streets have retained their inhabitants or have grown (Ufficio Satistica 2021, 1). Overlaying these streets over the diversity map, we find that growth almost always requires some level of diversity, as estimated by us (Fig. M2.).

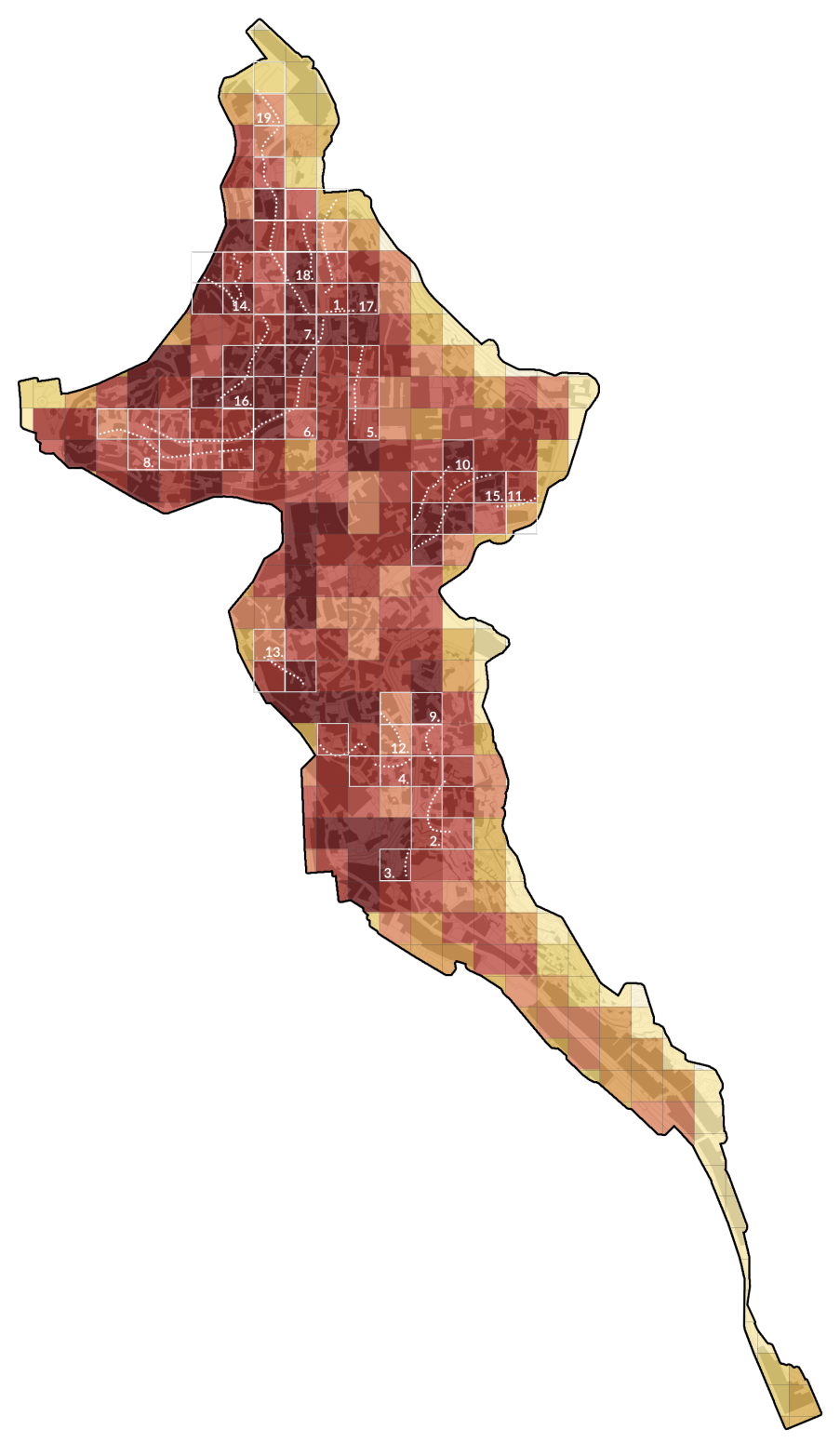

Figure M2. Validation of the diversity maps by studying diversification and growth. We consider census data from the period 1994-2019 (Ufficio Statistica 2021, 1). We locate streets that have experienced a stable or increased number of residents. We then overlay these streets over our diversity maps and evaluate all $6950 \times 50 \mathrm{~m}$ areas situated along these streets. 65 or $94 \%$ of these areas have a diversity score above 4 as displayed in the 2021 diversity map. Thus, we find an evident, positive correlation between diversity and the ability to attract or retain residents. Diversity is required for growth. Street names: 1.Via Casale. 2. Rione Casalnuovo. 3.Vico Casalnuovo. 4.Via Confalone. 5.Via Gradoni Duomo. 6.\& 7. Via Fiorentini. 8.Via Lombardi. 9. Rione Malve. 10.Via Muro. 11.Via Ospedale Vecchio. 12.Via del Purgatorio Vecchio. 13.Via San Bartolomeo. 14. Rione San Biagio. 15.Via San Giacomo. 16.Via San Giovanni Vecchio. 17. Gradelle San Nicola del Sole.

Another way to validate the diversity maps is by comparing diverse areas with urban centers, as represented on maps that are independent of ours. When defining diversity, we proposed that diversity is a defining characteristic of city. This means that diverse areas are expected to form urban centers. We overlay our map with urban centers as found on google maps. The four urban centers found on google maps coincide with places of sustained urban diversity in our maps (Fig. M3.). 


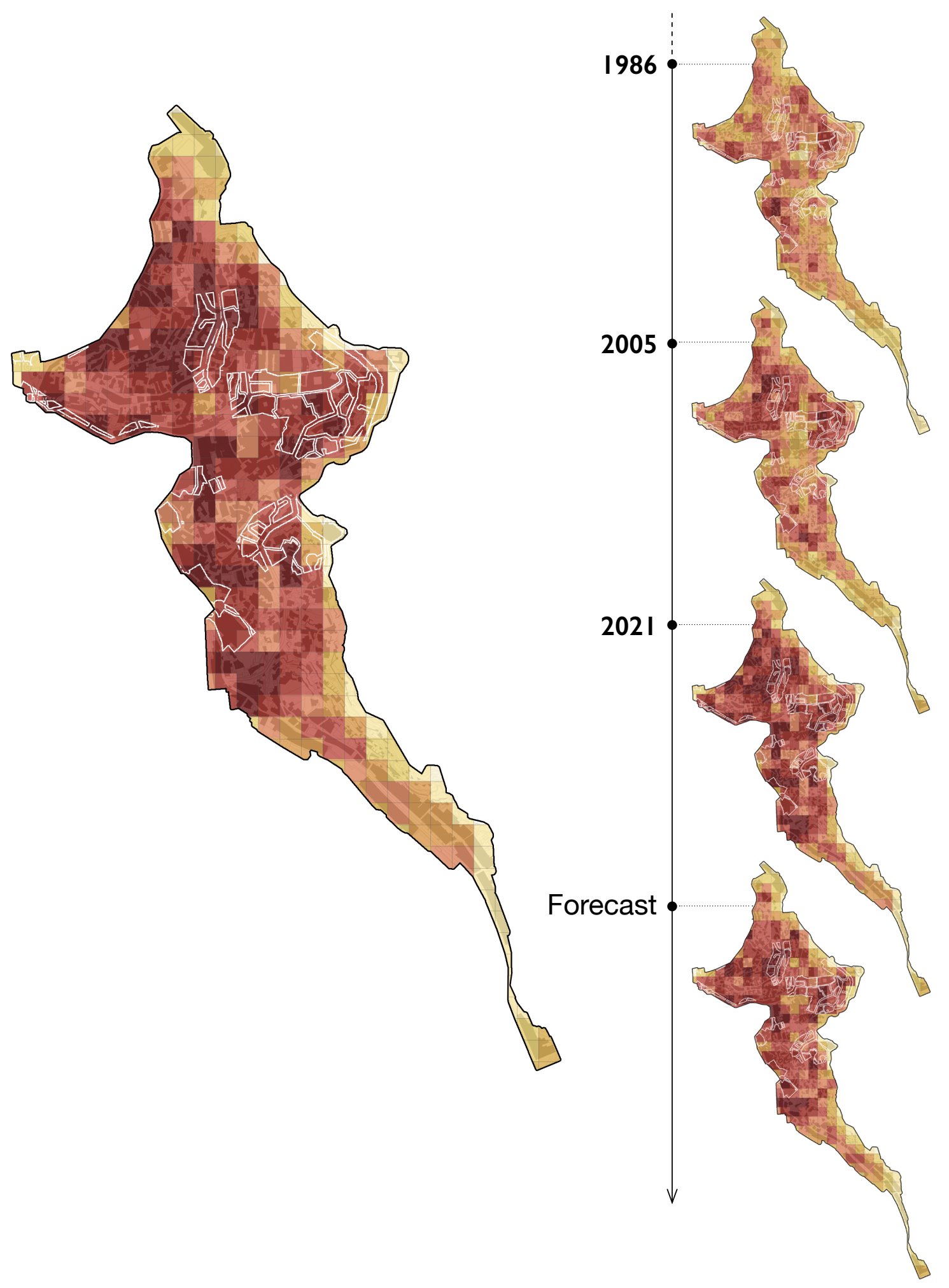

Figure M3. Validation through comparison of diversity with the locations of urban centers. Urban centers as found on google maps coincide well with places on our map that kept scoring high diversity. 
Finally, we can also validate the results through evaluation of historical photographs. This approach is displayed in Figure M4. We find that photographs that display high diversity, as defined in section 1, are located in areas that we ranked as diverse.
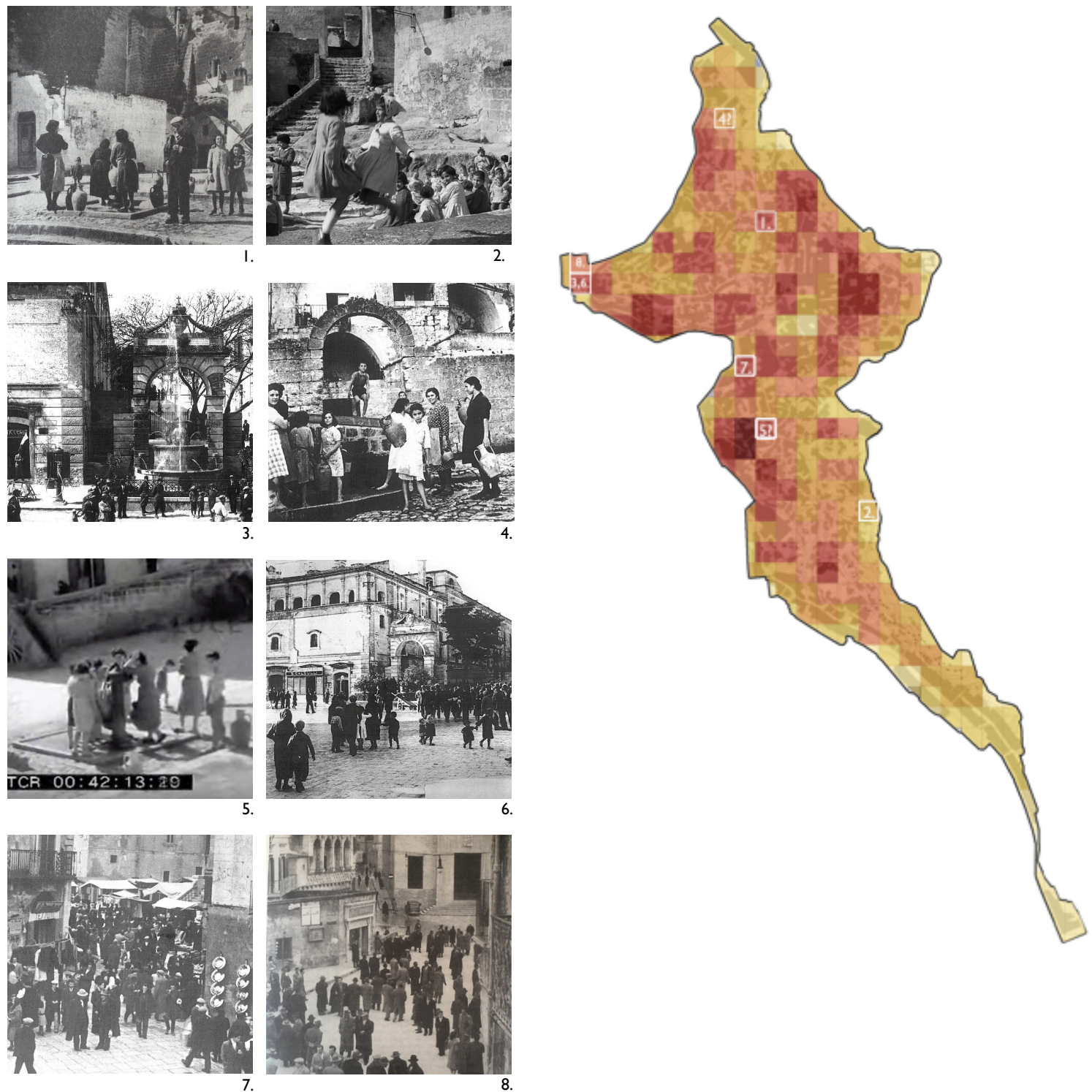

Figure M4. Validation of the diversity maps though historical photographs. The cataloged historical photographs, with exception of number 2 , are located in areas of diverse urban activity. Photograph number 2 was shot by Henri Cartier-Bresson along the stairs to the Malve cemetery. The picture shows activity, but only one party is involved. The diversity map of 1986 ranks these areas accordingly. The photographs are cataloged in chronological order. Location names: 1. Fountain in San Pietro Barisano Square. 2. Public stairs at Malve Cemetery. 3. Fountain Ferdinandea in Vittorio Veneto Square. 4. Fountain probably in Vico Santa Cesarea. 5. Fountain probably in Vico San Clemente. 6. Fountain Ferdinandea in Vittorio Veneto Square. 7. Sedile Square 8. Banco di Napoli in Vittorio Veneto Square. 
Next to Sassi, we have also mapped diversity in several modernist satellite settlements. We have obtained overall lower diversity scores in these settlements. Only small centers of diversity were observed. Let us now validate this result through historical evaluation. We shall very briefly consider how the modernist settlements were intended to function, how they were perceived historically, and how they have evolved.

When, in the 1950s, the inhabitants of Sassi were forcedly relocated to the modern suburbs, planners did wish to reproduce some of the urban qualities that were present in Sassi. To learn from Sassi, urban studies were led by Friedrich G. Friedmann (Friedman 1952; Musatti et al. 1996). However, it appears that the planners of the modernist settlements failed to comprehend the importance of urban diversity. Regrettably, the design of the new settlements was often led astray by a nostalgic and idealized vision of what planners called a 'vicinato,' or family neighborhood. Examples of family neighborhoods were sometimes found around fountains in Sassi, and their existence was often motivated by the antiquated social convention that women needed a neighbor's presence to go out. Already in the 1950s, some planners questioned the validity of the theory behind these family neighborhoods. One publication sarcastically asked, "How many urbanists and sociologists were led astray searching the Philosopher's Stone of the family neighborhood-which is an idealized ensemble of multiple families that are held together by no social bonds other than their common destiny as neighbors?" (Gorio, 1954, p. 139) Certainly, some planners did intend to transform the concept of the family neighborhood to create more elaborate community spaces, but these efforts, although ambitious in their scope, were often unsuccessful (De Carlo 1959).

Today, the modernist satellite settlements around Sassi are recognized as architecturally relevant examples of Italian Neorealism. Yet repeated episodes of vandalism are documented next to sporadic demolitions and years of neglected maintenance (Saito, 2020). It is also documented that some inhabitants of the modernist suburbs did return to Sassi. We interpret these observations as support for the thesis that diversity catalyzes growth while lack thereof may result in decay.

\section{Applications}

In our earlier methods-paper (Baciu, Birchall 2021), we have shown that our method to map urban diversity can be used to evaluate and re-evaluate diversity in both real and simulated settings. We have also predicted the existence of cycles of diversification, although we did not have sufficient empirical data to test our predictions. Our present research article and MethodsX paper provide these data. Yet, theory and data are most valuable if they can be used in meaningful ways. Therefore, we shall also propose actual applications for our maps, methods, and theory:

First, investors and architects can apply our method to estimate which of their planned interventions most contribute to diversification. Investors should be interested to support diversification because diversification leads to growth, which is 
what investors benefit from, in the first place. Investors profit well if they buy a property and this property gains value through interventions carried out after the property was purchased.

Second, authorities can on their end apply our method to estimate which planned interventions or entire urban strategies most contribute to diversification. Authorities should be interested in achieving diversification in cases in which it is needed because authorities, too, depend on the support of the population, which benefits from urban diversification in those cases. For example, authorities depend on tax money, which depends on property value and economic growth.

Third, without any particular intervention in mind, an early warning system can be set up to monitor low levels not only of growth but also of diversity. This warning system should be beneficial because it would allow authorities and citizens to act early, leveling cycles of diversification before decay begins.

Fourth, the warning system could be coupled to a forecast obtained through modeling growth and diversification through the replicator-mutator-equation or some other related mathematics. It should be valuable to monitor and forecast cycles of diversification and growth to inform the population. Businesses, landlords, and tenants who get the forecast can then decide whether or not they wish to get prepared.

Finally, our method can be used to formulate law that can be unambiguously translated into localized action, as we proposed in the research article. This application is beneficial because law works well when it is the same everywhere, while urban environments are spatially complex and evolve. What we propose is to set strict or tentative goals for diversity and growth. Setting such goals has the benefit of being independent of industry, time, and place. Our method brings the benefit that it allows to formulate such universal goals and translate them into local interventions. If our method and theory are followed, there is no more need to support specifically one or another industry in one place, oppose the same industry in another, and change the law all the time to cope with the tides of the cycle of diversification.

\section{Bibliography}

Baciu DC (2020). "Cultural life: Theory and empirical testing." BioSystems 197.104208. Baciu DC, Birchall C (2021). "Mapping Diversity: From Ecology and Human Geography to Urbanism and Culture." OSF preprints.

Commune di Matera $(2005,1)$. "Mappa delle tipologie architettoniche $\mathrm{A}_{1}$." [Map of architectonic types.] Retrieved from: https://www.comune.matera.it/images/sassi regolamenti/TAV A1 Tipologie 83.pdf

Commune di Matera $(2005,2)$. "Mappa delle attività $\mathrm{R}_{2}$." [Map of activities.] Retrieved from: https://www.comune.matera.it/images/sassi regolamenti/TAV R2 Attivit 83.pdf

Commune di Matera $(2005,3)$. " $1^{\circ}$ e $2^{\circ}$ Programma biennale di interventi per la conservaztione ed il recupero dei Rioni Sassi.” [Biennial program for interventions in the conservation and preservation of the Rioni Sassi.] Retrieved from: 
http://www.comune.matera.it/images/amm trasp/disposizioni generali/atti generali/regolamenti/rg NtaL771 NR.pdf [This is the technical documentation accompanying the legal document Commune di Matera 2005, 4. It provides an account of the urban types found in 1986 and 2005.]

Commune di Matera $(2005,4)$. "Previsioni generali del recupero in attuazione della 1. 11/11/1986 N $\mathrm{N}^{\circ} 771$ : Relazione." [General scheme for preservation in accord to law 11/11/1986 No 771.] Retrieved from: https://www.comune.matera.it/images/sassi_regolamenti/All_A_Relazione_83.pdf

De Carlo G (1959). "Il risultato di un concorso." [The result of a competition] Casabella continuità 231: 11-12. Retrieved April 14, 2021 from http://www2.unibas.it/architettura/CORSI/LOSASSO/Casabella\%20Continuita n 231 1959.pdf

Fisher RA, AS Corbet, CB Williams (1943). "The Relation Between the Number of Species and the Number of Individuals in a Random Sample of an Animal Population." J. Animal Ecol., 12, 42.

Friedmann FG (1952). "Osservazioni sul mondo contadino dell'Italia meridionale." Quaderni di sociologia [Observations on the rural life of Southern Italy", Notebooks of sociology] 26-27: 13-26. Accessed on April 14, 2021 from https://journals.openedition.org/qds/1590

Gorio F (1954). "Il Villaggio La Martella: autocritica." [La Martella village: a selfcritique] Casabella continuità 200. accessed on April 14, 2021 from https://ricerca.unich.it/retrieve/handle/11564/687076/123978/Matera

Hirschman AO (1945). National power and the structure of foreign trade. Berkeley: University of California Press.

Musatti R, FG Friedmann, et al (1996). Matera 55. Radiografia di una città del Sud [Matera 55. Radiography of a city in the South.]

Saito M (2020) Press Release. Do.Co.Mo.Mo Italia March 18. 2020.

Toxey A (2011). Materan Contradictions: Architecture, Preservation and Politics (Ashgate Studies in Architecture). Ashgate Publishing Group.

Ufficio Statistica Commune di Matera $(2021,1)$. "Movimento Demografico al 31 dicembre di ogni anno dal 1931 al 2017" [Demographic trend on December 31 for every year from 1931 to 2017.] Catalogo dei dati aperti. [Catalog of open data.] Retrieved from: http://dati.comune.matera.it/dataset/movimento-demografico-annuale-1931-2017-della-citta-di-matera

Ufficio Statistica Commune di Matera $(2021,2)$. "RNC Rilevazione Numeri Civici ed Edifici." [Survey of House Numbers and Buildings.] Catalogo dei dati aperti. [Catalog of open data.] Retrieved from: http://dati.comune.matera.it/dataset/rncrilevazione-numeri-civici-ed-edifici

\section{Data Repository}

The following data have been archived at DOI: 10.17605/OSF.IO/NWKPZ as a zip archive CD-Sassi_202105115.zip and will be made publicly available upon publication.

- DWG maps of Sassi

○ M1986.DWG Sassi 1986 from Commune di Matera 2005, 1 (data from official document created by the City of Matera) 
○ M2005.DWG Sassi 2005 from Commune di Matera 2005, 2 (data from official document created by the City of Matera)

o UT1986.DWG Sassi 1986 our urban types map (Our 1986 urban types map)

○ UT2005.DWG Sassi 2005 our urban types map (Our 2005 urban types map)

○ UT2021.DWG Sassi 2021 our urban types map (Our 2021 urban types map)

○ UTFC.DWG Sassi hypothetical forecast, urban types map (Our hypothetical forecast urban types map)

- GM.DWG Sassi granularity map, for all years 1986, 2005, and 2021 (Granularity map marking each distinct unit counted towards Simpson's diversity index)

- PDF maps of Sassi and Satellite Settlements

- M1986.PDF Sassi 1986 from Commune di Matera 2005, 1 (data from official document created by the City of Matera)

- M2005.PDF Sassi 2005 from Commune di Matera 2005, 2 (data from official document created by the City of Matera)

- UT1986.PDF Sassi 1986 our urban types map (Our 1986 urban types map)

○ UT2005.PDF Sassi 2005 our urban types map (Our 2005 urban types map)

- UT2021.PDF Sassi 2021 our urban types map (Our 2021 urban types map)

- UTFC.PDF Sassi hypothetical forecast, urban types map (Our hypothetical forecast urban types map)

- GM.PDF Sassi granularity map (Granularity map marking each distinct unit counted towards Simpson's diversity index)

- G.PDF Sassi google map of urban centers 2021 (Screenshot of google map with yellow areas)

○ MS.PDF satellite settlements, original plans

- PDF documentation

○ M2005-3.PDF Commune di Matera 2005, 3

○ M2005-4.PDF Commune di Matera 2005, 4.

- Tables

- Table1 Ufficio Statistica Commune di Matera 2021, 1 (Census data)

- Table2 Ufficio Statistica Commune di Matera 2021, 2 (Survey of Building Numbers and Buildings)

- Table3 Effective numbers counted towards Simpson's diversity index, over the entire period 1986-2021 with hypothetical forecast.

- Table4 Counts of buildings based on other sources and at different granularities, listed by year and source.

- PDF Diversity Maps

○ DM1986.PDF Sassi 1986

○ DM2005.PDF Sassi 2005

○ DM2021.PDF Sassi 2021

- DMFC.PDF Sassi hypothetical forecast

- DMMS.PDF satellite settlements 
The figures contain the following data:

- Figure 2 (in research article)

o our urban types maps 1986, 2005, 2021, and hypothetical forecast

- diversity maps 1986, 2005, 2021, and hypothetical forecast

○ classification of urban types

- analysis procedure scheme

- Simpson's index calculated from Table 3

- Figure 3 (in research article)

o Diversity Maps of satellite settlements

- Equation 1 (in MethodsX paper)

○ Table3 with computed Simpson's index 1/D.

- Figure M1 (in MethodsX paper)

○ excerpt of urban types map 1986, with sample areas in resolution and analysis grid

- $\quad$ Figure M2 (in MethodsX paper)

○ Diversity Map 2021, overlaid with streets that have retained their inhabitants or grown according to data in Table 1.

- $\quad$ Figure M3 (in MethodsX paper)

○ Diversity Maps 1986, 2005, 2021, and hypothetical forecast overlaid with G.PDF, Google map of urban centers

- Figure M4 (in MethodsX paper)

○ Diversity Map 1984, overlaid with locations where historical photographs (cataloged on the left) were taken

\section{Contributions}

Both authors contributed equally to this research. Dan C. Baciu has written the MethodsX paper. Diana Della Pietra has studied urban diversity and created the diversity maps of Matera as student under the lead of Dan C. Baciu as Assistant Professor of Digital Tools at TU Delft, Faculty of Architecture and the Built Environment.

\section{Identifiers}

Project DOI: 10.17605/OSF.IO/NWKPZ 